\title{
CORRELATION OF MEAN PLATELET VOLUME IN PATIENTS WITH CARDIAC SYNDROME X IN INDIAN POPULATION
}

\author{
Ram Anil Raj ${ }^{1}$, Yogesh Kothari², Kshamata Bewoor ${ }^{3}$, Sushant Kumar Sharma4, Venkataramaiah 5 \\ ${ }^{1}$ Assistant Professor, Department of Cardiology, Rajarajeshwari Medical College \& Hospital, Bangalore. \\ ${ }^{2}$ Associate Professor, Department of Cardiology, Rajarajeshwari Medical College \& Hospital, Bangalore. \\ ${ }_{3}^{3}$ Senior Resident, Department of Cardiology, Rajarajeshwari Medical College \& Hospital, Bangalore. \\ ${ }^{4}$ Senior Resident, Department of Cardiology, Rajarajeshwari Medical College \& Hospital, Bangalore. \\ 5 Professor and HOD, Department of Cardiology, Rajarajeshwari Medical College \& Hospital, Bangalore.
}

\section{ABSTRACT}

Platelets play a pivotal role in the development of atherosclerotic lesions, plaque destabilization and atherothrombosis. The methods of testing platelet activity can be time-consuming, expensive and technically difficult. Unlike more expensive or timeconsuming methods of assessing platelet function, the determination of platelet size by quantification of Mean Platelet Volume (MPV), using automated haemograms, is simple and inexpensive. "Cardiac syndrome X" characterized with 1. Angina or angina-like chest pain, 2. ST segment depression that can be induced by treadmill exercise testing, 3. Normal coronary arteriography. As cardiac syndrome $\mathrm{X}$ and angina pectoris caused by typical obstructive coronary heart disease should be distinguished from each other for effective treatment. Although there has not been a clear explanation of the exact pathophysiological mechanism underlying cardiac Syndrome X, studies are suggestive that coronary micro-circular abnormalities and endothelial dysfunction play a role in the aetiology of the disease. Atherosclerosis and endothelial vasomotor dysfunction have been suggested as possible contributing factors. There are limited datas in literature to compare the MPV in cardiac syndrome X. This study was designed to compare MPVs of patients with Cardiac syndrome X, patient with CAD and of normal controls.

\section{METHODS}

Nine hundred and forty four patients who had undergone coronary angiography between May 2014 and April 2016 in Rajarajeshwari Medical College and Hospital, Bangalore, were retrospectively examined. Of these, 118 patients were enrolled in the study and were divided into three groups. The Cardiac Syndrome X group consisted of 40 subjects (18 men and 22 women with mean age group $45+4.2$ years). The CAD group consisted of 33 subjects (18 men and 15 women with mean age of $46+4.8$ years) with CAD, which was defined as $>50 \%$ stenosis in a minimum of one coronary artery. The control group consisted of 45 age and sex matched individuals ( 23 men and 22 women with mean age of $45+4.5$ years) who presented with chest pain, normal coronaries and no ischaemia during treadmill exercise testing.

\section{RESULTS}

The primary characteristics of the study population are given in Table 1 . There was no statistical significance between the three groups with respect to age, sex, smoking or alcohol consumption ( $p=N S)$. There were no statistically significant differences in the MPV measurements between the cardiac syndrome X group and CAD groups. (Mean MPV 12.2 $\pm 1.4 \mathrm{fL} \mathrm{vs.} 13.8 \pm 2.1 \mathrm{fL}, \mathrm{p}=\mathrm{NS}$ ). The MPV was significantly higher in both Cardiac syndrome X (mean $=12.2 \pm 1.4 \mathrm{fL})$ and CAD group (mean $=13.8 \pm 2.1 \mathrm{fL})$ compared with the control groups (mean $=7.9 \pm 1.1 \mathrm{fL})(\mathrm{p}<0.01)$.

\section{CONCLUSION}

Elevated MPVs may participate in the pathogenesis of Cardiac Syndrome X. Prospective, placebo-controlled studies with large sample sizes that use multivariable survival analyses and long-term follow-up periods are required for the clinical evaluation of the prognostic value of increased MPV in patients with Cardiac Syndrome X.

\section{KEYWORDS}

Cardiac Syndrome X, Mean Platelet Volume, Coronary Artery Disease.

HOW TO CITE THIS ARTICLE: Raj RA, Kothari Y, Bewoor K, et al. Correlation of mean platelet volume in patients with cardiac syndrome X in Indian population. J. Evolution Med. Dent. Sci. 2016;5(62):4323-4326, DOI: 10.14260/jemds/2016/987

\section{INTRODUCTION}

Platelets play a pivotal role in the development of atherosclerotic lesions, plaque destabilization and athero-

Financial or Other, Competing Interest: None.

Submission 11-07-2016, Peer Review 23-07-2016,

Acceptance 26-07-2016, Published 02-08-2016.

Corresponding Author:

Dr. Ram Anil Raj,

Assistant Professor,

Department of Cardiology,

Rajarajeshwari Medical College \& Hospital,

No. 202, Kambipura, Mysore Road,

Bengaluru-560074.

E-mail: drramanilraj2001@yahoo.com

DOI: $10.14260 /$ jemds $/ 2016 / 987$ thrombosis. ${ }^{1}$ The methods of testing platelet activity can be time-consuming, expensive and technically difficult. ${ }^{2}$ Unlike more expensive or time-consuming methods of assessing platelet function, the determination of platelet size by quantification of Mean Platelet Volume (MPV) using automated haemograms is simple and inexpensive. 3 The biologic rationale linking MPV to clinical outcomes along with its universal availability has made it a promising indirect marker of platelet reactivity. MPV is a marker of platelet size and activity and a predictor of cardiovascular risk. ${ }^{4}$

Kemp. 5 in 1973 coined a term "cardiac syndrome X" with the following three characteristics: 1. Angina or angina-like chest pain, 2. ST segment depression that can be induced by 
treadmill exercise testing, 3. Normal coronary arteriography. As cardiac syndrome $\mathrm{X}$ and angina pectoris caused by typical obstructive coronary heart disease should be distinguished from each other for effective treatment. In diagnosis of noncardiac origin such as oesophageal disorders or psychiatric condition should be considered and ruled out. Although, there has not been a clear explanation of the exact pathophysiological mechanism underlying cardiac syndrome $\mathrm{X}$, studies suggestive that coronary micro-circular abnormalities and endothelial dysfunction play a role in the aetiology of the disease. Atherosclerosis and endothelial vasomotor dysfunction have been suggested as possible contributing factors. ${ }^{6-8}$

There are limited data in literature to compare the MPV in cardiac syndrome $\mathrm{X}$. This study was designed to compare MPVs of patients with cardiac syndrome $X$, patient with CAD and of normal controls.

\section{METHODS}

Nine hundred and forty four patients who had undergone coronary angiography between May 2014 and April 2016 in Rajarajeshwari Medical College and Hospital, Bangalore, were retrospectively examined. Of these, 118 patients were enrolled in the study and were divided into three groups.

The cardiac syndrome X-group consisted of 40 subjects (18 men and 22 women with mean age group $45+4.2$ years). The diagnosis of cardiac syndrome $\mathrm{X}$ was based on the presence of typical exercise-induced angina pectoris that was associated with transient ischaemic ST segment depression $(>1 \mathrm{~mm})$ during the treadmill exercise testing with normal coronary angiogram in the absence of coronary artery spasm as determined by hyperventilation manoeuvre.

The CAD group consisted of 33 subjects (18 men and 15 women with mean age of $46+4.8$ years) with CAD, which was defined as $>50 \%$ stenosis in a minimum of one coronary artery.

The control group consisted of 45 age and sex matched individuals (23 men and 22 women with mean age of $45+4.5$ years) who presented with chest pain, normal coronaries and no ischaemia during treadmill exercise testing.

Written informed consent was obtained from all of the participants prior to enrolment. The Institutional Ethics Committee approved the study protocol.

All the subjects were evaluated with a detailed medical history, physical examination and biochemical analysis which included 12-hour fasting lipid profile (includes low density lipoprotein cholesterol, high density lipoprotein cholesterol and triglyceride). All of the subjects were questioned regarding cardiovascular drug use, smoking and alcohol consumption. All the subjects underwent transthoracic echocardiography for structural heart disease.

The exclusion criteria included the following: refusal to participate in the study and presence of hypertension, diabetes, left ventricular dysfunction (left ventricular ejection fraction $<50 \%$ or hypertrophy, unstable ischaemic condition (unstable angina pectoris and myocardial infarction), valvular heart disease, congenital heart disease, any observed abnormality in thyroid function tests, renal or hepatic dysfunction (Creatinine $>1.2 \mathrm{mg} / \mathrm{dL}$, aspartate aminotransferase and alanine transaminase levels that were higher than double the upper limits of normal, respectively), primary haematological disorder, inflammatory diseases, any infection within three months and any medication that could affect the MPV.

\section{Cardiac Catheterization}

The coronary angiograms were performed with a radial approach using the TIG catheter with the use of heparin 2500 units, 100 microgram of Nitroglycerin and 100 microgram of diltiazem. Following the appropriate patient preparation, the entire patient in the study population underwent elective coronary artery arteriography using Siemens Catheterization lab. Two experienced cardiologists who were blinded to the study subject's group assignment evaluated all of the angiograms. The coronary angiograms were judged with regards to a smooth appearance, luminal wall irregularities, Epicardial local or diffuse caliber reduction or stenosis. The coronary arteries were classified as normal on the basis of visual assessment of the absence of any luminal irregularities. To exclude the possibility of coronary artery vasospasm, the patient who exhibited normal coronary anatomy underwent a hyperventilation test during the coronary arteriography. The hyperventilation test was performed by asking the patient to breathe rapidly and deeply for 5 minutes.

\section{Biochemical Measurement}

The blood samples were withdrawn without stasis on the morning of the day prior to the coronary angiography and following as fasting period of 12 hours. The glucose, creatinine, and lipid profiles were determined using standard methods. For all the groups, we measured the MPV from the blood samples that were obtained following the venepuncture. The blood was collected in tripotassium EDTA tubes. We analysed the blood samples of all of the groups using an automatic blood counter after two hours of venepuncture. This period of time was waited to allow for the stabilization of platelet shape changes.

\section{Statistical Analyses}

The continuous variables are reported as the mean \pm standard deviation, whereas the categorical variables were defined as percentages. The Statistical Package for the Social Sciences (SPSS) statistical software package version 15 for windows was used for the statistical analysis. Statistical significance was defined as $\mathrm{p}<0.05$.

\section{RESULTS}

The primary characteristics of the study population are given in Table 1. There was no statistical significance between the three groups with respect to age, sex, smoking or alcohol consumption ( $p=N S$ ). There was a female preponderance in cardiac syndrome $X$ group, but not statistically significant. The body mass indexes, lipid profiles and fasting glucose levels did not differ between the three groups. Likewise, the laboratory characteristics of all of the groups were not significantly different. There were no statistically significant differences in the MPV measurements between the cardiac syndrome $\mathrm{X}$ group and CAD groups. (Mean MPV 12.2 $\pm 1.4 \mathrm{fL}$ vs. $13.8 \pm 2.1 \mathrm{fL}$ $\mathrm{p}=\mathrm{NS}$ ). The MPV was significantly higher in both Cardiac syndrome $\mathrm{X}$ (mean=12.2 $\pm 1.4 \mathrm{fL}$ ) and CAD group (mean= $13.8 \pm 2.1 \mathrm{fL}$ ) compared with the control groups (mean $=7.9 \pm 1.1$ fL) $(\mathrm{p}<0.01)$. 


\begin{tabular}{|c|c|c|c|c|}
\hline & $\begin{array}{c}\text { Control Group } \\
(n=45)\end{array}$ & $\begin{array}{c}\text { Cardiac Syndrome } \\
\text { X Group }(n=40)\end{array}$ & $\begin{array}{c}\text { CAD Group } \\
(n=33)\end{array}$ & $\begin{array}{c}\mathbf{p} \\
\text { Valve }\end{array}$ \\
\hline Age, yrs. & $45.3 \pm 4.5$ & $45.3 \pm 5.1$ & $46.2 \pm 4.8$ & NS \\
\hline Gender (M), n (\%) & $23(51)$ & $18(45)$ & $18(54)$ & $\mathrm{NS}, \mathrm{p}=0.08$ \\
\hline Active smoker, \% (n) & $24.4(11)$ & $25(10)$ & $24(8)$ & NS \\
\hline Alcohol consumption, \% (n) & $11(5)$ & $10(4)$ & $9(3)$ & NS \\
\hline BMI (kg/m2) & $26.8 \pm 4.1$ & $27.9 \pm 5.6$ & $26.1 \pm 4.1$ & $\mathrm{NS}, \mathrm{p}=0.11$ \\
\hline Fasting blood glucose (mg/dL) & $99 \pm 9$ & $104 \pm 9$ & $100 \pm 11$ & NS \\
\hline Haemoglobin (mg/dL) & $12.8 \pm 1.3$ & $12.4 \pm 1.4$ & $12.2 \pm 1.3$ & NS \\
\hline LDL cholesterol (mg/dL) & $129.4 \pm 28.3$ & $134.6 \pm 24.3$ & $135.4 \pm 33.3$ & NS \\
\hline HDL cholesterol (mg/dL) & $35.4 \pm 8.9$ & $34.5 \pm 7.8$ & $34.2 \pm 6.7$ & NS \\
\hline Triglycerides (mg/dL) & $168.9 \pm 57.2$ & $170.4 \pm 60.2$ & $169.2 \pm 69.3$ & NS \\
\hline Creatinine $(\mathrm{mg} / \mathrm{dL})$ & $0.98 \pm 0.14$ & $1.07 \pm 0.22$ & $1.02 \pm 0.18$ & NS \\
\hline Platelet count (L/cumm) & $1.66 \pm 0.22$ & $1.64 \pm 0.44$ & $1.71 \pm 0.21$ & NS \\
\hline Mean platelet volume (fL) & $7.9 \pm 1.1$ & $12.2 \pm 1.4$ & $13.8 \pm 2.1$ & $P=0.01$ \\
\hline
\end{tabular}

Table 1: The Baseline Clinical and Biochemical Characteristics of the Study Groups

\section{DISCUSSION}

Patients with Cardiac Syndrome X have been stated to have multiple pathophysiological abnormalities. ${ }^{9}$ Generalized endothelial dysfunction and inflammation were reported as the most convincing evidence of cardiac syndrome X.10,11 Also in the patient with cardiac syndrome X; inflammation was thought to be related to endothelial dysfunction. Elevated levels of inflammatory molecules were previously reported as markers of atherosclerotic disease activity and as an indication of increased risk from the progression of atherosclerosis. ${ }^{12}$ Moreover, inpatients with either coronary artery disease or Cardiac Syndrome X, creative protein was reported to be associated with impaired endothelial function. CRP levels was found to be correlated with the activity of the disease. In a similar vein, elevated Red Cell Distribution Width (RDW) levels were found to be independently associated with presence of Cardiac Syndrome X, and in the same study RDW and CRP levels were reported to correlate positively. ${ }^{13}$ In another study, the presence of endothelial dysfunction and sub-angiographic atheroma was revealed in patients with Cardiac Syndrome X.14 Furthermore, some intravascular ultrasonographic studies with Cardiac Syndrome X patients discovered presence of abnormal coronary arteries with atheromatous plaques and intimal thickening. ${ }^{14,15}$ Additionally, magnetic resonance imaging revealed abnormal sub-endocardial perfusion in patients with Cardiac Syndrome $\mathrm{X} .{ }^{16}$ For all these reasons, it could be considered that the aetiopathogenesis of coronary artery disease and Cardiac Syndrome $\mathrm{X}$ are similar.

Platelets play an important role in the pathogenesis, morbidity and mortality of acute coronary syndromes.17 Increased platelet activation and aggregation were reported to be closely associated with cardiovascular complications. ${ }^{18}$ The average size of platelets is described as mean platelet volume. Larger platelets have been found to be biologically more active and their pro-thrombotic properties are more powerful.

In another study, the relationship between MPV and angiographic severity of coronary artery disease was investigated and a positive correlation between them was found. ${ }^{18}$ Cay et al documented elevated MPV in patients with acute coronary syndrome and CSX.19 Similar to our study, Demirkol et al ${ }^{20}$ showed that the MVP values were significantly higher in the Cardiac Syndrome X and coronary artery disease groups than those of the control group.
Platelet function can be affected by several factors such as platelet count, size, density, platelet age, a higher granule count and adhesion receptor expression as well as higher mean platelet volume. MPV appears to correlate more closely with platelet volume than does platelet count alone. ${ }^{21}$ Even though various methods like optical aggregometry, platelet function analysis using PFA-100 system, platelet reactivity tests, platelet aggregate ratio measurements, flow cytometry and thromboxane B2 generation tests 22 are there to analyse the platelet activation, but they are of limited use due to complex pre-analytical factors, reduced specificity and poor reproducibility. MPV is a simpler marker that does not require advanced or expensive technology. MPV may reflect platelet production and stimulation; therefore, elevated MPV value may indicate cardiovascular disease. Increased MPV may be due to the body's use of small platelets during acute ischaemia. ${ }^{23}$ Thus, MPV has become a prognostic factor in coronary heart disease and may eventually be accepted as a parameter of platelet activity. ${ }^{24}$ In addition, several reports have demonstrated that there is a close relationship between MPV and cardiovascular risk factors including impaired fasting glucose levels, diabetes mellitus, hypertension, hypercholesterolemia, obesity and metabolic syndrome. ${ }^{25-27}$ It has been reported that elevated MPVs are associated with cardiovascular diseases. ${ }^{28}$ It is also known that increased platelet activation and aggregation are closely related to cardiovascular complications. ${ }^{24}$

The primary limitation of our study was the comparatively small size of the study population. Another limitation was the possibility of underlying coronary artery spasm in patients with Cardiac Syndrome $\mathrm{X}$, which was excluded using a hyperventilation test, although the ergonovine test would have been the ideal test in this contest. Also, coronary flow reserve measurement using Doppler wire and intravascular ultrasound are two invasive methods that could be used in the diagnosis of microvascular disease and exclusion of atheromatous plaques on the coronary vessel wall, respectively. Positron emission tomography might be used for detection of abnormalities in the coronary vasomotor functions caused by microvascular disease and to measure the coronary flow reserve. Moreover, perfusion abnormalities caused by coronary microvascular disease could be reliably detected by contrast stress echocardiography and cardiac magnetic resonance, which are non-invasive assessments. 
None of these tests were performed in our study subjects, who were assessed retrospectively in this study.

\section{CONCLUSION}

MPV was significantly higher in patients with either Cardiac Syndrome X or Coronary Artery Disease relative to the control, but with no statistically significant differences were observed between the Cardiac Syndrome $\mathrm{X}$ and the CAD groups. Elevated MPVs may participate in the pathogenesis of Cardiac Syndrome X. Prospective, placebo-controlled studies with large sample sizes that use multivariable survival analyses and long-term follow-up periods are required for the clinical evaluation of the prognostic value of increased MPV in patients with Cardiac Syndrome X.

\section{REFERENCES}

1. Davì G, Patrono C. Platelet activation and atherothrombosis. N Engl J Med 2007;357:2482-94.

2. Michelson AD. Methods for the measurement of platelet function. Am J Cardiol 2009;103(Suppl 3):20A-26.

3. Boos CJ, Lip GY. Assessment of mean platelet volume in coronary artery disease-what does it mean? Thromb Res 2007;120(1):11-3.

4. Chu SG, Becker RC, Berger PB, et al. Mean platelet volume as a predictor of cardiovascular risk: a systematic review and meta-analysis. J Thromb Haemost 2010;8(1):148-56.

5. Kemp HG. Left ventricular function in patients with the anginal syndrome and normal coronary arteriograms. Am J Cardiol 1973;32(3):375-6.

6. Egashira K, Inou T, Hirooka Y, et al. Evidence of impaired endothelium-dependent coronary vasodilatation in patients with angina pectoris and normal coronary angiograms. N Engl J Med 1993;328(23):1659-64.

7. Quyyumi AA, Cannon RO, Panza JA, et al. Endothelial dysfunction in patients with chest pain and normal coronary arteries. Circulation 1992;86(6):1864-71.

8. Vane JR, Anggard EE, Botting RM. Regulatory functions of the vascular endothelium. N Engl J Med 1990;323(1):2736.

9. Al Suwaidi J, Higano S, Holmes DR, et al. Pathophysiology, diagnosis, and current management strategies for chest pain in patients with normal findings on angiography. Mayo Clin Proc 2001;76(8):813-22.

10. Hurst T, Olson TH, Olson LE, et al. Cardiac syndrome $x$ and endothelial dysfunction: new concepts in prognosis and treatment. Am J Med 2006;119(7):560-6.

11. Demirkol S, Balta S, Unlu $M$, et al. Neutrophils/lymphocytes ratio in patients with cardiac syndrome $\mathrm{x}$ and its association with carotid intima-media thickness. Clin Appl Thromb Hemost 2014;20(3):250-5.

12. Lind L. Circulating markers of inflammation and atherosclerosis. Atherosclerosis 2003;169(2):203-14.

13. Qing P, Luo SH, Guo YL, et al. Evaluation of red blood cell distribution width in patients with cardiac syndrome $\mathrm{x}$. Dis Markers 2013;34(5):333-9.

14. Cox ID, Clague JR, Bagger J, et al. Endothelial dysfunction, subangiographic atheroma, and unstable symptoms in patients with chest pain and normal coronary arteriograms. Clin Cardiol 2000;23(9):645-52.
15. Wiedermann JG, Schwartz A, Apfelbaum M. Anatomic and physiologic heterogeneity in patients with syndrome $\mathrm{x}$ : an intravascular ultrasound study. J Am Coll Cardiol 1995;25(6):1310-7.

16. Panting JR, Gatehouse PD, Yang GZ, et al. Abnormal subendocardial perfusion in cardiac syndrome $\mathrm{x}$ detected by cardiovascular magnetic resonance imaging. $\mathrm{N}$ Engl J Med 2002;346(25):1948-53.

17. Thaulow E, Erikssen J, Sandvik L, et al. Blood platelet count and function are related to total and cardiovascular death in apparently healthy men. Circulation 1991;84(2):613-7. http://dx.doi.org/ 10.1161/01.CIR.84.2.613.

18. Ekici B, Erkan AF, Alhan A, et al. Is mean platelet volume associated with the angiographic severity of coronary artery disease? Kardiol Pol 2013;71(8):832-8.

19. Cay S, Biyikoglu F, Cihan G, et al. Mean platelet volume in the patients with cardiac syndrome $\mathrm{x}$. J Thromb Thrombolysis 2005;20(3):175-8.

20. Demirkol S, Balta S, Unlu M, et al. Evaluation of the mean platelet volume in patients with cardiac syndrome $\mathrm{x}$. Clinics (Sao Paulo) 2012;67(9):1019-22.

21. Thompson CB, Jakubowski JA. The pathophysiology and clinical relevance of platelet heterogeneity. Blood 1988;72(1):1-8.

22. Haubelt $\mathrm{H}$, Simon $\mathrm{M}$, Anders $\mathrm{CH}$, et al. Platelet function tests for monitoring of acetylsalicylic acid: clinical significance in antiplatelet treatment. Hamostaseologie 2004;24(3):196-202.

23. Sewell R, Ibbotson RM, Phillips R, et al. High mean platelet volume after myocardial infarction: is it due to consumption of small platelets? Br Med J (Clin Res Ed) 1984;289(6458):1576-8. http://dx.doi.org/10.1136/bmj.289.6458.1576.

24. Erhart S, Beer JH, Reinhart WH. Influence of aspirin on platelet count and volume in humans. Acta Haematol 1999;101(3):140-4. http://dx.doi.org/10.1159/000040940.

25. Tavil Y, Sen N, Yazici HU, et al. Mean platelet volume in patients with metabolic syndrome and its relationship with coronary artery disease. Thromb Res 2007;120(2):245-50. http://dx.doi.org/10.1016/j.thromres.2006.10.005.

26. Varol E, Akcay S, Ozaydin M, et al. Mean platelet volume is associated with insulin resistance in non-obese, nondiabetic patients with coronary artery disease. J Cardiol 2010;56(2):154-8.

27. Coban E, Bostan F, Ozdogan M. The mean platelet volume in subjects with impaired fasting glucose. Platelets 2006;17(1):67-9. http://dx.doi.org/10.1080/09537100500220729.

28. Jagroop IA, Mikhailidis DP. Mean platelet volume is an independent risk factor for myocardial infarction but not for coronary artery disease. $\mathrm{Br} \mathrm{J}$ Haematol 2003; 120(1):169-70. 